\title{
Survey on Content based Image Retrieval to Deal with Rapid Growth of Digital Images
}

\author{
Nitish Barya \\ Department of Electronics and Communication, \\ Truba Institute of Engineering and Information \\ Technology, Bhopal
}

\author{
Himanshu Jaiswal \\ Department of Electronics and Communication, \\ Truba Institute of Engineering and Information \\ Technology, Bhopal
}

\begin{abstract}
Development in image retrieval systems has increased in large part due to the rapid growth of the digital images produced by World Wide Web and high capacity of digital data storage devices available in the human race domain. A desired image from network and storage media is shared by citizens belonging to various field, including education, business, government agencies, journalism, and advertising agencies. But due to generation of large collection of digital images, users are not satisfied with the traditional information retrieval techniques. As the elaboration of multimedia technologies are becoming more trendy, so these days the content based image retrieval are becoming a foundation of exact and fast retrieval. This survey paper deals with the techniques of content based image retrieval using both local features and global features. One of them is conventional color histogram and fuzzy color histogram. Further Support vector machine (SVM) with optimized feature sub set selection using radial bias network can also be used to improve retrieval performance.
\end{abstract}

\section{General Terms}

Content Based Image Retrieval.

\section{Keywords}

Color Histogram, Content Based Image Retrieval, Fuzzy color histogram and Segmentation

\section{INTRODUCTION}

Progress in computer and network tools fasten with relatively cheap high capacity digital data storage devices have brought incredible growth in the amount of digital images that are currently widely used in medicine, fashion, crime prevention, face recognition, finger print recognition and bio-metrics etc. Hence, efficient image searching and retrieval system is significant. The approach is based on two aspects of image research (IR): text-based retrieval and content-based retrieval. Text-Based Approach has some evident faults. As different people categorize the same image differently so it is difficult for a new person to retrieve the image categorize by some other person. Conventional techniques allow for textual searches on Meta information only. Content Based Image Retrieval (CBIR) is a technique to avoid these problems that in standard helps to sort out digital image by their visual content. In past years, many CBIR systems have been created, the common argument for them is to extract a desired image. Comparing two images and checking whether they are similar or not is a comparatively easy thing to do for a human. Getting a computer to accomplish

The same thing effectively is a different issue .Content Based Image Retrieval (CBIR) is a system which employs image contents to search images from large image database by using query image. To extract low level features Firstly the texture feature can be compute using contrast, energy, correlation, and homogeneity functions. Then the color feature can be calculated by using color moments such as color expectancy, color variance and skewness. Lastly, shape feature can be calculated using edge detection operators like sobel, prewitt etc. The same measurement attributes can be used to evaluate both local and global features. Fuzzy color histogram is one of the appropriate technique of image retrieval and further SVMRBF (Radial basis function) can be used to reduce the semantic gap and improve the retrieval performance.

\section{LITERATURE SURVEY}

This section gives an extensive literature survey on the existing content based image retrieval method based on features descriptor and optimization technique. In 2004, Issam El-Naqa, Yongyi Yang, Nikolas P. Galatsanos, Robert M. Nishikawa , and Miles N. Wernick [1] suggested a learning machine-based structure for illustrating human intuitive resemblance for CBIR. A unique mechanism was proffered by Hiremath P.C.[2] in 2005 for image retrieval with the help of shape, color and shape features. Salient points based on color saliency are computed on the images. From the fixed sized windows the texture and color features are been obtained, around the salient points, to perform as local descriptors. To obtain the edge images of objects the gradient vector flow fields are used. To narrate the shape features invariant moments are used. A union of these local color, texture and global shape characteristics provides a rstrong set of features for image retrieval. A suitable image retrieval pattern was proffered in 2006 by Zhe-Ming Lu, Su-Zhi Li and Hans Burkhardt [10] in DCT domain that is suitable for retrieval of different size of color JPEG images. In 2007, Ryszard S. Chora's [6] identifies the problems existing in CBIR and Biometrics systems - describing image content and image feature extraction. He has described a possible approach to mapping image content onto low-level features. This paper investigated the use of a number of different color, texture and shape features for image retrieval in CBIR and Biometrics systems.In 2007, Ch.Srinivasa Rao, S. Srinivas kumar \& B.N.Chatterji [8] describes the CBIR system with rotational invariance. In 2008 , S. Nandagopalan, Dr. B. S. Adiga, [12] proposed a universal model for the Content Based Image Retrieval System by combining the color, texture, and edge density features or individually.In 2009, Hichem Bannour_Lobna ,Hlaoua_Bechir Ayeb, [7] proposed two methods of content based image retrieval according to visual similarity. The first method consists in indexing the images automatically through global features calculated on the whole image, while the second consists in indexing the image using features calculated on the regions of the image. In 2010, H. B. Kekre \& Dhirendra Mishra, [4] presented a new algorithm for 
digital image search and retrieval. They have used Fast Fourier Transform of each R, G and B component of images separately. Also in 2010, H.B. Kekre [9] presented two algorithms with the application of Kekre's transform and conclude that even though the database is having variety of images still the system is performing better in terms of precision for both the approaches. In 2010, Jalil Abbas, Salman Qadri, Muhammad Idrees, Sarfraz Awan and Naeem Akhtar Khan, [5] focuses on both the content based image retrieval and Text Based image retrieval (TBIR). In 2010, Ramesh Babu Durai [11] shows that the outcome of the results using DCT for feature extraction is pretty promising.

In 2011, R. Joe [1] describe automatic image retrieval for clinical image database. Full text searching is performed to find evidence in support of a clinical decision. In this research, image analysis and classification techniques are explored to automatically extract information for differentiating specific modalities to characterize illustrations in biomedical publications, which may assist in the evidence finding process.

D. N. F. AwangIskandar James A. Thom S. M. M. Tahaghoghi[13] describe that a common approach to contentbased image retrieval is to use example images as queries. Images in the collection that have low-level features similar to the query examples are returned in response to the query. Chih-Chin Lai and Ying-Chuan Chen [15] proposed a useroriented mechanism for CBIR method based on an interactive genetic algorithm (IGA). In 2011, Ming Qi, Guangzhong Sun and Guoliang Chen [16] describe feature extraction for content based image retrieval based on parallel features optimization. They describe both thread-parallel optimization and Single Instruction Multiple Data (SIMD) optimizations and used SIMD logical operations to eliminate the random conditional branches which cannot be effectively predicted by CPU Branch Prediction.In April 2012, M. Subrahmanyam, R.P. Maheshwari and R. Balasubramanian [17] describe content based image retrieval based on expert system and new technique of image indexing and retrieval system. A new image indexing and retrieval system for content based image retrieval (CBIR) is proposed in this title. The characteristics (vector points) of image are computed using color (color histogram) and SOT (spatial orientation tree).

In 2012, Heng Chen and Zhicheng Zhao [3] describe relevance feedback method for image retrieval. Relevance feedback (RF) is an effective method for content-based image classification (CBIC), and it is also a feasible step to shorten the semantic gap between low-level visual feature and highlevel perception. SVM-based RF algorithm is proposed to improve performance of image classification.

\section{MOTIVATION}

At the present time, content-based image retrieval (CBIR) has become research spotlight of multimedia Information retrieval. Usually, low-level features of images, such as color, texture, and shape, are used to illustrate images and to measure the content similarity between two images. However the conventional query method is easy to implement, CBIR is still unapproachable for common people owing to the dissatisfactory query result. Resemblance measure is a key constituent in image retrieval. Conventionally, Euclidean distances are used to measure the similarity between the query and the images in the database. The smaller distance, more similar the database image to the query. Though this metric is sensitive to the sample topology. The Euclidean distancebased similarity measure can be examined as sketch a hyper- sphere in the high-dimensional feature space, centered at particular point .The greater the radius of the hyper-sphere, the more images is surrounded in the hyper-sphere.The radius is calculated indirectly by the number of retrieved images. One probable scheme to identify the boundary is support vector machine. Though, the SVM classifier generally requires a large number of examples to calculate approximately the model parameters, which is not practical for image retrieval because we cannot anticipate the users to propose many examples in the interactive process. Instead, we choose learning methods that are non-parametric and do not require a huge number of examples to find out a decision boundary.

\section{CONTENT BASED IMAGE RETRIEVAL SYSTEM}

Please use a 9-point Times Roman font, or other Roman font CBIR systems search collection of images based on features that can be extracted from the image files themselves without manual descriptive. The objective of CBIR systems is to support image retrieval based on content e.g., shape, color, texture. In past decades many CBIR systems have been developed, the common ground for them is to extract a desired image. Comparing two images and deciding if they are similar or not is a relatively easy thing to do for a human. Getting a computer to do the same thing effectively is however a different matter.

The algorithms used in these systems are commonly divided into three errands: extraction, selection, and classification. The extraction mission is to converts wealthy content of images into various content features. Feature extraction is the procedure of generating features to be used in the selection and classification tasks. Feature selection decreases the number of features provided to the classification task. Those features which are likely to help in bias are selected and used in the classification task. Features which are not selected are discarded. Of these three activities, feature extraction is most critical because the particular features made available for discrimination directly influence the efficacy of the classification task. The end result of the extraction task is a set of features, commonly called a feature vector, which constitutes a representation of the image. In the last few decades, a number of above revealed techniques of image content feature extraction confirmed reliable enough for practical applications in industrial automation, biomedicine, social security, biometric authentication and crime prevention.

\section{IMAGE FEATURE}

For pages other than the first page, start at the top of the page, there are basically two type of feature local or global. If the features are extracted from the illustration of the entire image, then these features are called global features. Generally global features are used successfully for image retrieval. The main difficulty that is linked with the global features is that the resulting retrieved images cannot differentiate between the object of interest and the background. Therefore in compare to global features, it is necessary to extract only local features from regions of concern or objects in the image. But again there is some problem with using local feature is that it required image segmentation to determine the regions of interest

\subsection{Texture}

In the field of computer dream and image processing there is no appropriate definition of texture because existing texture 
definitions are based on texture analysis techniques. Texture is a prime component of human visual perception.

\subsection{Color}

One of the most significant feature of image that make promising the recognition of images is color. Color is a property that depends on the reflection of light to the eye and the processing of that information in the brain. Naturally, the color of an image is represented through some color model. There exist various color models to depict color information. A color model is 3-Dimentional coordinate system used for representing color in terms of intensity values. Generally used color models are RGB(red, green, blue),HSV(hue, saturation, value) and $\mathrm{Y}, \mathrm{Cb}, \mathrm{Cr}$ (luminance and chrominance).

\subsection{Shape}

Shape feature can be calculated using edge detection operators like sobel, prewitt etc. It may also be achieved by using $\mathrm{EDH}($ Edge histogram descriptor).The edge histogram descriptor represents the spatial distribution of five types of edges, say four directional edges and one non-directional edge. As edges play a vital role for image perception, it can retrieve images with similar meaning. Thus, it gives good results for natural images with non-uniform edge distribution.

\section{COLOR HISTOGRAM}

One of the most popular way of representation of color content of the image is by using color histogram. Statistically, it indicates the frequency of occurrence of every color in an image. For a three-dimensional image, three of such histograms can be plotted. The histograms are generally divided into bins to roughly represent the content and reduce complexity of succeeding matching stage. For image retrieval, histogram of query images is compared with the histogram of all images in the database using some similarity metric.

Because conventional color histogram illustrates the distribution of colors within the entire or within an interest region of image. Thus histogram is invariant to rotation, translation and scaling of an object but the histogram does not hold semantic information, and two images with similar color histograms can exhibit different contents.

There are several complications associated with the conventional color histogram $(\mathrm{CH})$ as mentioned above. To handle the difficulty of rotation and translation, an invariant color histograms based on the color gradients can be adopt which make use of gradients in dissimilar channels that weight the influence of a pixel on the histogram to finsh out the changes produced by deformations. To sort out the problem of spatial relationship and large differences between neighboring bins of conventional color histograms, fuzzy color histogram $(\mathrm{FCH})$ can be used, by considering the color similarity of each pixel's color related to all the histogram bins through fuzzy-set membership function. Instead of assigning each pixel into one of the bins only as in conventional color histogram $(\mathrm{CCH})$, FCH extends each pixel's total membership value to all the histogram bins.

\section{SUPPORT VECTOR MACHINE- RADIAL BASIS FUNCTION}

Do not include headers, footers or page numbers in your Support vector machines (SVMs, also called support vector networks) are supervised learning models with linked learning algorithms that analyze data and identify patterns, used for image retrieval and classification. Known a set of training examples, each marked as belonging to one of two categories, an SVM training algorithm constructs a model that assigns new examples into one category or the other, making it a nonprobabilistic binary linear classifier. An SVM model is a representation of the examples as points in space, mapped so that the examples of the separate categories are divided by a clear gap that is as wide as possible. New examples are then mapped into that same space and predicted to belong to a category based on which side of the gap they fall on. In addition to performing linear classification, SVMs can efficiently perform a non-linear classification using what is called the kernel trick, implicitly mapping their inputs into high-dimensional feature spaces.

For the classification of lower content of image support vector machine (SVM) is generally used. Machine Learning is measured as a subfield of Artificial Intelligence and it is concerned with the development of techniques and methods which enable the computer to learn. In simple terms development of algorithms which enable the machine to learn and perform tasks and activities. Machine learning overlaps with statistics in many ways.

Image feature selection process decides the performance of image retrieval. The optimized feature sub set selection ca be perform using radial bias network. The output of RBF network proceed input for support vector machine classifier. The basic idea is to carry out the training process of the hidden layer of RBF neural classifiers by taking into account the class-memberships of the training samples. In exacting, clusters are generated by grouping training samples belonging to the same class in order to avoid the formation of mixed clusters.

\section{PROBLEM STATEMENT}

This review paper covers various research and journal papers related to content based image retrieval system. Most of the papers faced same problem i.e. semantic gap between query image and retrieval image. The semantic gap is the lack of concurrence between the content that one can extract from the visual data and the understanding that the same data have for a user in a given situation. User seeks semantic similarity, but the database can only provide similarity by data processing.

Large amount of time is also required to search desired image among database images. It is also observed that images with different perceptions are also be found similar by some retrieval systems.

\section{CONCLUSION}

In this review paper, we focused on a survey of different techniques of content based image retrieval to improve the performance of image retrieval system. Also, this paper gives a review of the works carried out, in eliminating the semantic gap between the low level features and the high level semantic concepts. Fuzzy color histogram is one of the appropriate method of image retrieval over conventional color histogram method and further SVM- RBF (Radial basis function) can be used to reduce the semantic gap and improve the retrieval performance. But due to creation of vast amount of digital images, users are not satisfied with the current image retrieval techniques. Hence further improvement is required to improve the quality of image retrieval. A key aspect of this survey is to suggest a system that, in addition to combine the use of existing low level features with new feature to match images that may give better result.

\section{REFERENCES}

[1] R.Joe Stanleya Soumya Dea, Dina Demner-Fushmanb, Sameer Antanib, George R. Thomab "An image feature- 
based approach to automatically find images for application to clinical decision support". Computer Med Imaging Graph. 2011 Jul; 35 (5):365-72.

[2] Hiremath P.S. and Jagadeesh Pujari "Content Based Image Retrieval using Color Boosted Salient Points and Shape features of an image" International Journal of Image Processing, Volume (2) : Issue (1).

[3] Heng Chen and Zhicheng Zhao "An effective relevance feedback algorithm for image retrival"inIEEE 2012. This paper appears in:Network Infrastructure and Digital Content (IC-NIDC), 2012 3rd IEEE International Conference.

[4] Dr. H. B. Kekre, Dhirendra Mishra "CBIR using Upper Six FFT Sectors of Color Images for Feature Vector Generation" H.B.Kekre. et al /International Journal of Engineering and Technology Vol.2(2), 2010, 49-54.

[5] Jalil Abbas, Salman Qadri, Muhammad Idrees, Sarfraz Awan, Naeem Akhtar Khan1 "Frame Work for Content Based Image Retrieval (Textual Based) System" Journal of American Science 2010; 6(9).

[6] Ryszard S. Chora's "Image Feature Extraction Techniques and Their Applications for CBIR and Biometrics Systems" international journal of biology and biomedical engineering, 2007.

[7] Hichem Bannour_Lobna Hlaoua_Bechir Ayeb, "Survey of the Adequate Descriptor for Content Based Image Retrieval on the Web: Global Versus Local Features "2009.

[8] Ch.Srinivasa rao , S. Srinivas kumar \#, B.N.Chatterji “ Content Based Image Retrieval using Contourlet Transform" ICGST-GVIP Journal, Volume 7, Issue 3, November 2007

[9] Dr. H. B. Kekre, Dhirendra Mishra "CBIR using Upper Six FFT Sectors of Color Images for Feature Vector Generation" H.B.Kekre. et al /International Journal of Engineering and Technology Vol.2(2), 2010, 49-54.

[10] Zhe-Ming Lu, Su-Zhi Li and Hans Burkhardt , “ A Content-Based Image Retrieval Scheme in Jpeg
Compressed Domain " International Journal of Innovative Computing, Information and Control ICIC International ${ }^{\circ} \mathrm{C} 2006$ ISSN 1349-4198 Volume 2, Number 4, August 2006

[11] Ramesh Babu Durai C “A Generic Approach To Content Based Image Retrieval Using Det And Classification Techniques" (IJCSE) International Journal on Computer Science and Engineering Vol. 02, No. 06, 2010, 20222024.

[12] S. Nandagopalan, Dr. B. S. Adiga, and N. Deepak "A Universal Model for Content-Based Image Retrieval" World Academy of Science, Engineering and Technology 462008

[13] D. N. F. AwangIskandar James A. Thom S. M. M Tahaghoghi "Content-based ImageRetrieval Using Image Regions as Query Examples" 9th conference on Australasian database, Volume 75, Pages 38-46.

[14] Issam El-Naqa, Yongyi Yang, Nikolas P. Galatsanos , Robert M. Nishikawa, and Miles N. Wernick , "A Similarity Learning Approach to Content-Based Image Retrieval: Application to Digital Mammography " Ieee Transactions On Medical Imaging, Vol. 23, No. 10, October 2004

[15] Chih-Chin Lai, Member, IEEE, and Ying-Chuan Chen "A User-Oriented Image Retrieval System Based on Interactive Genetic Algorithm”. IEEE Transaction on Instrumentation and Measurement, Volume 60, Issue 10 Pages 3318-3325.

[16] Ming Qi, Guangzhong Sun and GuoliangChen"Parallel and SIMD Optimization of Image Feature Extraction" International Conference on Computational Science, ICCS 2011.

[17] M. Subrahmanyam, R.P. Maheshwari and R Balasubramanian"Expert system design usingwavelet and color vocabulary trees for image retrieval, Expert Systems with ApplicationsVolume 39, Issue 5, April 2012, Pages 5104-5114. 